\title{
Identification of key genes and pathways associated with spinal cord injury
}

\author{
YUE-HUI ZHANG, JIA SONG, LI-GANG WANG and JIANG SHAO \\ Department of Orthopedic Surgery, Xin Hua Hospital Affiliated to \\ Shanghai Jiao Tong University School of Medicine, Shanghai 200092, P.R. China
}

Received December 14, 2015; Accepted December 19, 2016

DOI: $10.3892 / \mathrm{mmr} .2017 .6192$

\begin{abstract}
The present study was designed to identify key genes or significant signaling pathways associated with spinal cord injury (SCI), and to clarify the underlying molecular mechanisms of SCI. Data from the GSE45550 array were downloaded from the Gene Expression Omnibus database. A total of 6 control samples, 6 samples at 3 days post-SCI (SCI3d), 6 samples at 8 days post-SCI (SCI8d) and 6 samples at 14 days post-SCI (SCI14d) were included. The microarray data was preprocessed by the robust multi-array average algorithm. The differentially expressed genes (DEGs) were identified using the limma package. The overlapping DEGs among groups were analyzed using the Venny 2.0 online tool. Modules enriched by DEGs were selected by weighted gene co-expression network analysis. Gene Ontology annotation and the Kyoto Encyclopedia of Genes and Genomes pathways were identified for DEGs using the Database for Annotation, Visualization and Integrated Discovery. A total of 693 genes were obtained by combining the DEGs of the SCI3d, SCI8d and SCI14d groups. The pink module and green module with smaller P-values obtained from weighted gene co-expression network analysis module analyses of DEGs demonstrated a higher correlation with SCI. In addition, the peroxisome proliferator-activated receptor (PPAR) signaling pathway that the cluster of differentiation 36 (CD36) was significantly enriched in, was one of the significant pathways in the pink module. The p53 signaling pathway that Caspase-3 (Casp3) was significantly enriched in was one of the significant pathways in the
\end{abstract}

Correspondence to: Dr Jiang Shao, Department of Orthopedic Surgery, Xin Hua Hospital Affiliated to Shanghai Jiao Tong University School of Medicine, 1665 Kongjiang Road, Shanghai 200092, P.R. China

E-mail: shaojxh@126.com

Abbreviations: Casp3, Caspase-3; DEGs, differentially expressed genes; $C D 36$, cluster of differentiation 36; KEGG, Kyoto Encyclopedia of Genes and Genomes; PPAR, peroxisome proliferator-activated receptor; SCI, spinal cord injury

Key words: spinal cord injury, differentially expressed genes, venn diagram analysis, weighted correlation network analysis, pathways green module. In conclusion, the results of the present study demonstrated that the PPAR and p53 signaling pathway may serve important roles in the progression of SCI. In addition, CD36 and Casp3 may be involved in the progression of SCI via the PPAR and p53 signaling pathways, respectively.

\section{Introduction}

Spinal cord injury (SCI) is an injury to the spinal cord caused by trauma or disease, which may lead to alterations to the normal motor, sensory or autonomic function of the spinal cord (1). SCI is associated with high morbidity and mortality rates, and the SCI annual incidence rate ranges between 12.1-57.8 cases/million individuals $(1,2)$. In addition, the epidemiology of SCI is variable in different countries, and there is currently no effective treatment $(3,4)$. Therefore, an effective therapy for the treatment of SCI is required, and it has been suggested that genes associated with SCI may be able to provide novel strategies for such a treatment.

Despite the lack of effective treatments, there have been some notable findings associated with the molecular mechanism of SCI. It has been observed that SCI results in secondary degeneration involving apoptosis, with an increased expression of genes associated with apoptosis and decreased expression of anti-apoptotic genes (5). In addition, a reduction in excessive M1 macrophage polarization and an enhancement of M2 macrophage polarization produced by regulating the levels of cytokines, including tumor necrosis factor a and interleukin (IL)- $1 \beta$ in the SCI microenvironment, may be a desirable treatment method (6). Tachibana et al (7) observed that 3 genes, including heat shock $27 \mathrm{kDa}$ protein, tissue inhibitor of metalloproteinase-1 and epidermal fatty acid-binding protein, were upregulated in SCI. A recent study indicated that deletion of the IL-1 $\alpha$ gene protected oligodendrocytes from SCI by overexpressing TOX high mobility group box family member 3 (8). Furthermore, numerous genes associated with inflammation, such as Arginase 1, are differentially expressed in the ephrin type A-receptor 4 knockout mouse model of SCI (9). In addition, it has been demonstrated that a temporal blockade of the IL-6 signaling pathway may modify the inflammatory response following SCI, and thus promote regeneration of the spinal cord (10). However, there are various additional important genes and pathways associated with SCI that have yet to be explored completely. Thus, a greater understanding 
of these genes and pathways is required as they may provide novel targets for SCI therapy.

In the present study, GSE45550 microarray data was obtained from the Gene Expression Omnibus (GEO) and used to identify the differentially expressed genes (DEGs) associated with SCI. Functional enrichment analyses were performed for DEGs. Furthermore, functions of gene modules were analyzed. The aim of the present study was to identify critical genes or significant signaling pathways associated with SCI, and clarify the underlying molecular mechanisms involved.

\section{Materials and methods}

Affymetrix microarray data. The microarray data from GSE45550 was downloaded from the GEO database (http://www.ncbi.nlm.nih.gov/geo/) (11). The following 4 groups were applied: 6 control samples, 6 samples at 3 days post-SCI (SCI3d), 6 samples at 8 days post-SCI (SCI8d) and 6 samples at 14 days post-SCI (SCI14d). Data from the GPL1355 platform [(Rat230_2) Affymetrix Rat Genome 230 2.0 Array; Affymetrix Inc., Santa Clara, CA, USA] were used for subsequent analysis.

Data preprocessing. The microarray data was preprocessed using the robust multi-array average algorithm with the Affy package (12) in Bioconductor (version 1.46.1; http://www. bioconductor.org/). Background correction, normalization and calculation of expression were all included in the process of preprocessing. The probe of the microarray data was transformed to gene symbols with Bioconductor AnnotationData software packages. If several probes were mapped to one gene symbol, then the mean value was set as the final expression value of this gene. A total of 18,634 gene expression matrixes were obtained from the above process.

DEGs analysis. The DEGs in the following three comparison groups: SCI3d vs. Control, SCI8d vs. Control and SCI14d vs. Control were analyzed using the limma package (13) in Bioconductor. The DEG P-values were calculated using the unpaired Student's t-test (14) provided by the limma package, and the P-values were adjusted to false discovery rate (FDR) values using the Benjamini-Hochberg correction (15). $\log _{2}$ fold-change $(F C) \geq 1$ and FDR values $<0.05$ were used as cut-off criterion for DEGs. Hierarchical clustering analysis of the DEGs was then performed and visualized using g-plots (16) in the $\mathrm{R}$ package.

Venn diagram analysis of DEGs. Venny is an interactive tool used to compare lists with Venn diagrams (17). The Kyoto Encyclopedia of Genes and Genomes (KEGG; www.genome. $\mathrm{jp} / \mathrm{kegg} /$ ) database is used to put associated gene sets into their respective pathway (18). The Database for Annotation, Visualization and Integrated Discovery (DAVID; http://david. ncifcrf.gov), used for analyzing gene lists, is an integrated data-mining environment (19).

The intersections of upregulated and downregulated genes in different sample groups were respectively analyzed using Venny 2.0 (17) (http://bioinfogp.cnb.csic. es/tools/venny/index.html) online tool. KEGG pathway enrichment analysis was performed for the intersection of genes by DAVID. $\mathrm{P} \leq 0.05$ and gene counts $\geq 2$ were used as threshold values.

Analysis of the correlation between gene modules and phenotype. Weighted gene co-expression network analysis (WGCNA) (20) is a tool used to identify gene clusters or modules which are highly associated with the phenotype of samples in expression profile data, and generalize module characteristic genes among these gene clusters. Furthermore, WGCNA provides correlation coefficients and significant thresholds in every module.

In the present study DEGs in the SCI3d, SCI8d and SCI14d groups were combined, and the correlation between these DEGs and SCI3d, SCI8d and SCI14d were analyzed, and gene sets with higher correlation were dug. Modules enriched by DEGs were selected by WGCNA in the R package, and modules significantly associated with SCI were identified with cluster analysis. The higher the absolute value of the correlation coefficient, the closer the correlation was between gene expression levels in modules and SCI.

Enrichment analysis of module function. Gene Ontology (GO) is a tool used to generate gene annotations by collecting defined, structured and controlled vocabulary (21). GO annotation and KEGG pathway analyses were performed for DEGs using DAVID. $\mathrm{P}<0.05$ and gene counts $\geq 2$ were set as threshold values.

\section{Results}

Normalized analysis of sample data. The boxplots of sample data prior to and following normalization are depicted in Fig. 1. The median line of the boxplot was at the same level following normalization, indicating that all data were successfully normalized.

$D E G$ analysis. The DEG count of the three SCI groups compared with the control group are summarized in Table I with $\log _{2} \mathrm{FCl}$ values $\geq 1$ and FDR values $<0.05$. The heat maps of the DEGs are depicted in Fig. 2.

Analysis of overlapping DEGs among groups. Venn diagram analyses for the upregulated and downregulated genes in the SCI3d, SCI8d and SCI14d groups were performed, and overlapping DEGs among groups is depicted in Fig. 3. A total of 9 genes were upregulated at all 3 time points and 48 genes were downregulated at all 3 time points.

Where $\mathrm{P}<0.05$, there was no significant enrichment observed among the 9 intersecting upregulated genes using KEGG pathway analysis. However, the 48 intersecting downregulated genes were markedly enriched in the peroxisome proliferator-activated receptor (PPAR) signaling pathway $\left(\mathrm{P}=8.01 \times 10^{-4}\right.$; enriched genes including lipoprotein lipase, fatty acid binding protein 3, aquaporin 7 and adiponectin, C1Q and collagen domain containing) and in the synthesis and degradation of ketone bodies signaling pathway $\left(\mathrm{P}=2.39 \times 10^{-2}\right.$; enriched genes including 3-oxoacid CoA transferase 1, 3-hydroxybutyrate dehydrogenase, type 1) (data not shown). 
Table I. Differentially expressed genes count relative to the control group.

\begin{tabular}{lccc}
\hline Group & $\begin{array}{c}\text { Upregulated } \\
\text { genes count }\end{array}$ & $\begin{array}{c}\text { Downregulated } \\
\text { genes count }\end{array}$ & Total \\
\hline SCI3d & 232 & 322 & 554 \\
SCI8d & 121 & 142 & 263 \\
SCI14d & 64 & 71 & 135 \\
\hline
\end{tabular}

SCI3d, samples taken at 3 days post-SCI; SCI8d, samples taken at 8 days post-SCI; SCI14d, samples taken at 14 days post-SCI.
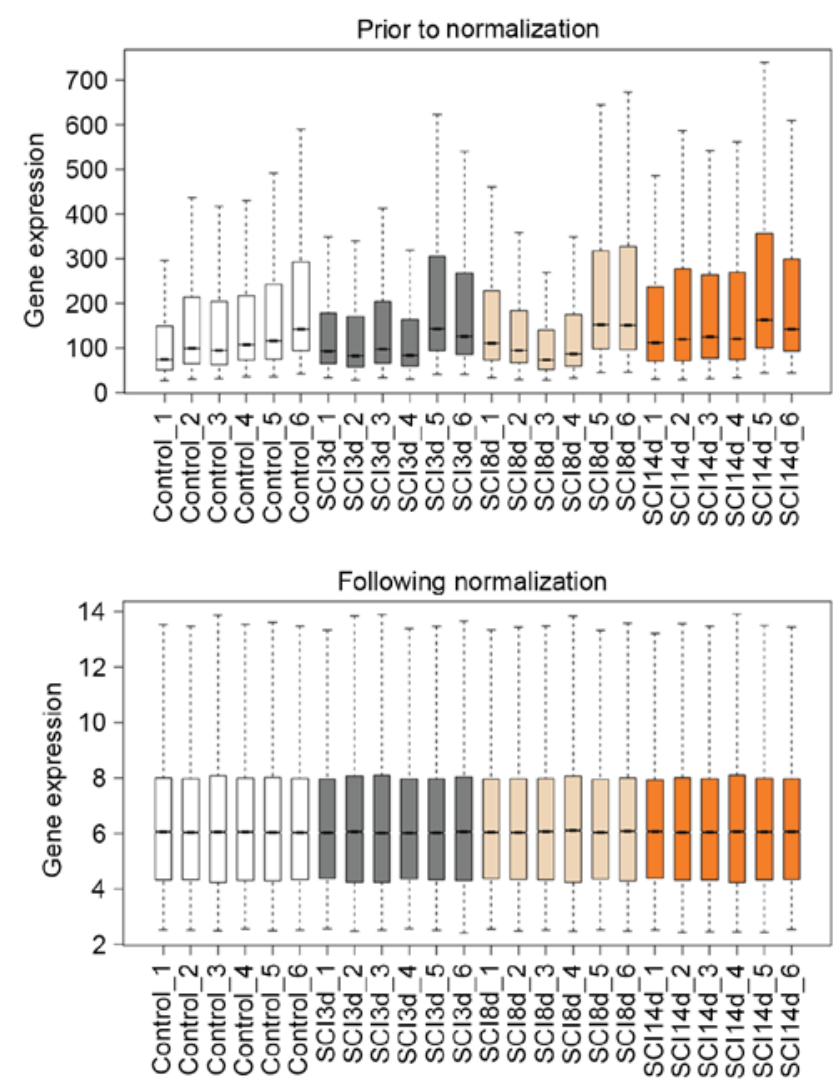

Figure 1. Boxplots of sample data prior to and following normalization. The $\mathrm{x}$-axis represents the sample names, and the $\mathrm{y}$-axis represents gene expression levels following $\log _{2}$ transformation. SCI, spinal cord injury; SCI3d, samples taken at 3 days post-SCI; SCI8d, samples taken at 8 days post-SCI; SCI14d, samples taken at 14 days post-SCI.

WGCNA module analyses of DEGs. A total of 693 genes were obtained by combining the DEGs of the SCI3d, SCI8d and SCI14d groups. Cluster analyses using WGCNA were performed using the expression data of these genes, and the cluster dendrogram is presented in Fig. 4. The DEGs were divided into 7 modules (pink module, green/yellow module, black module, blue module, green module, red module and tan module). The correlation coefficient and P-value between gene counts of every module and SCI are summarized in Table II. The data indicated that the pink module and green module with smaller P-values demonstrated a higher correlation with SCI. The functional enrichment results of pink and green

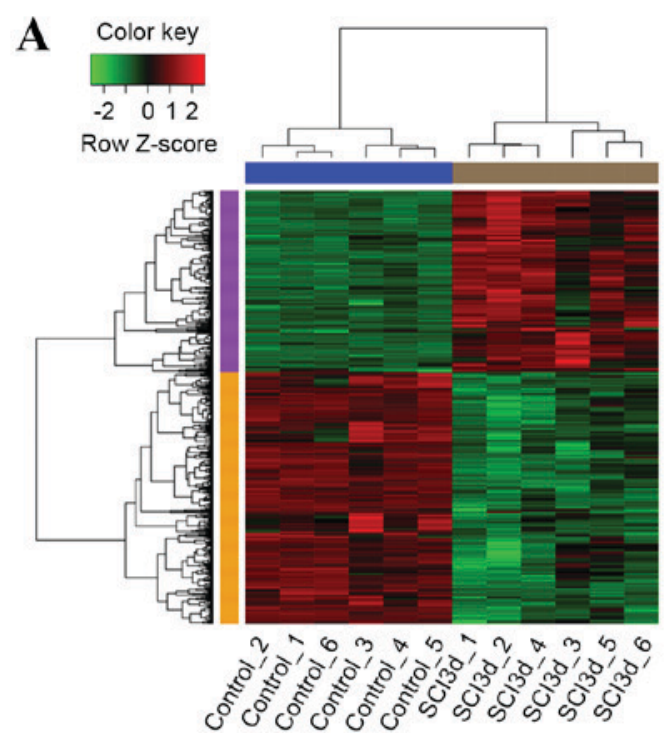

B
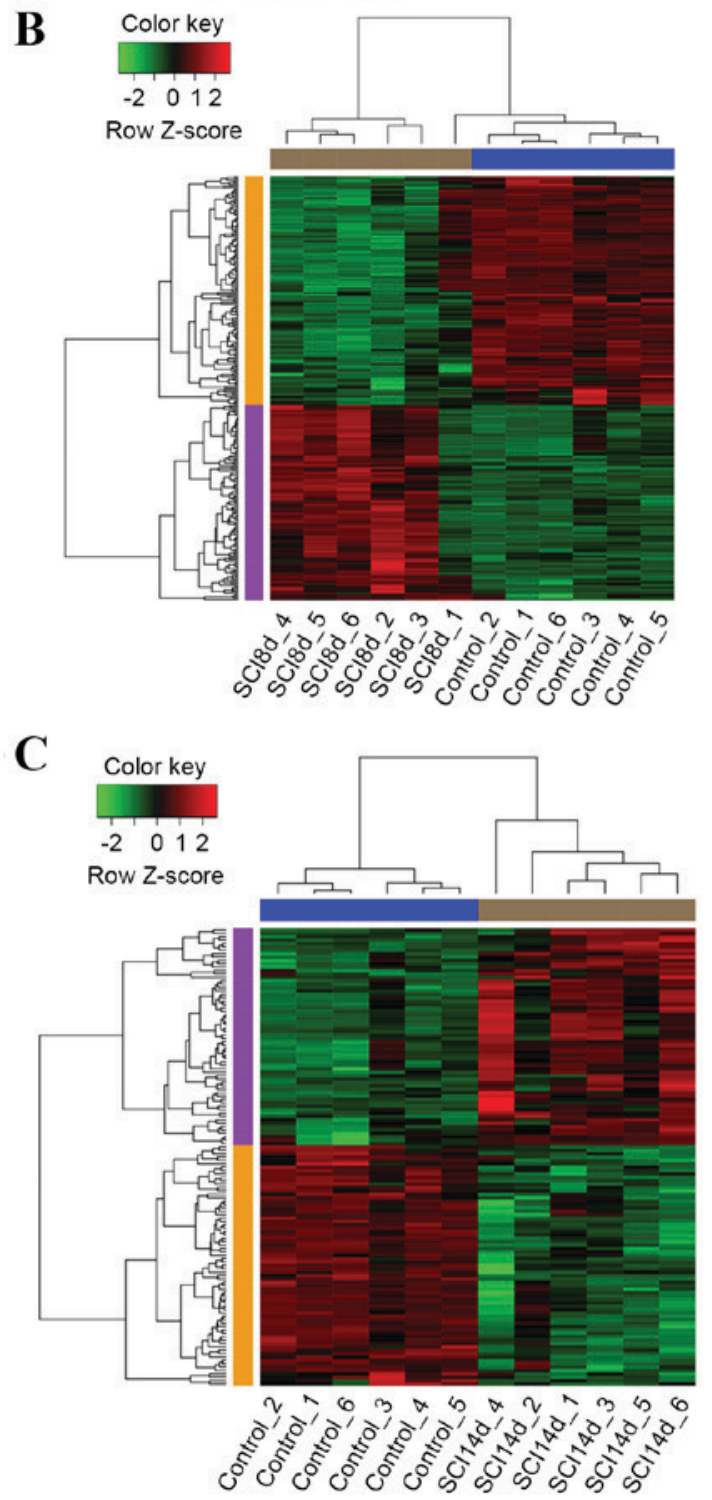

Figure 2. Heat maps of the DEGs in the (A) SCI3d group, (B) SCI8d group and (C) SCI14d group relative to the control group. The green represents reduced expression levels, red represents higher expression levels and black indicates no differential expression among genes. DEGs, differentially expressed genes; SCI, spinal cord injury; SCI3d, samples taken at 3 days post-SCI; SCI8d, samples taken at 8 days post-SCI; SCI14d, samples taken at 14 days post-SCI. 

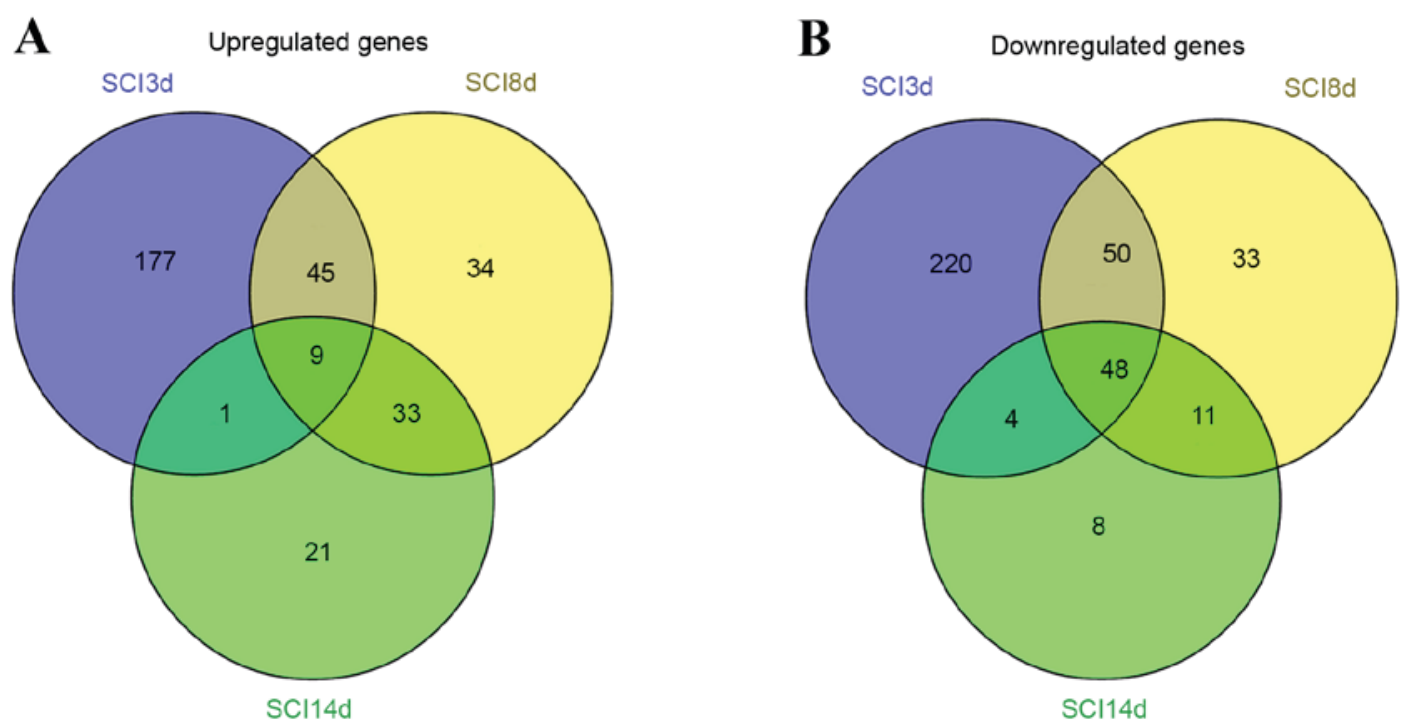

Figure 3. Intersectioning (A) upregulated and (B) downregulated genes in the SCI3d, SCI8d and SCI14d groups relative to controls. SCI, spinal cord injury; SCI3d, samples taken at 3 days post-SCI; SCI8d, samples taken at 8 days post-SCI; SCI14d, samples taken at 14 days post-SCI.

\section{Cluster Dendrogram}

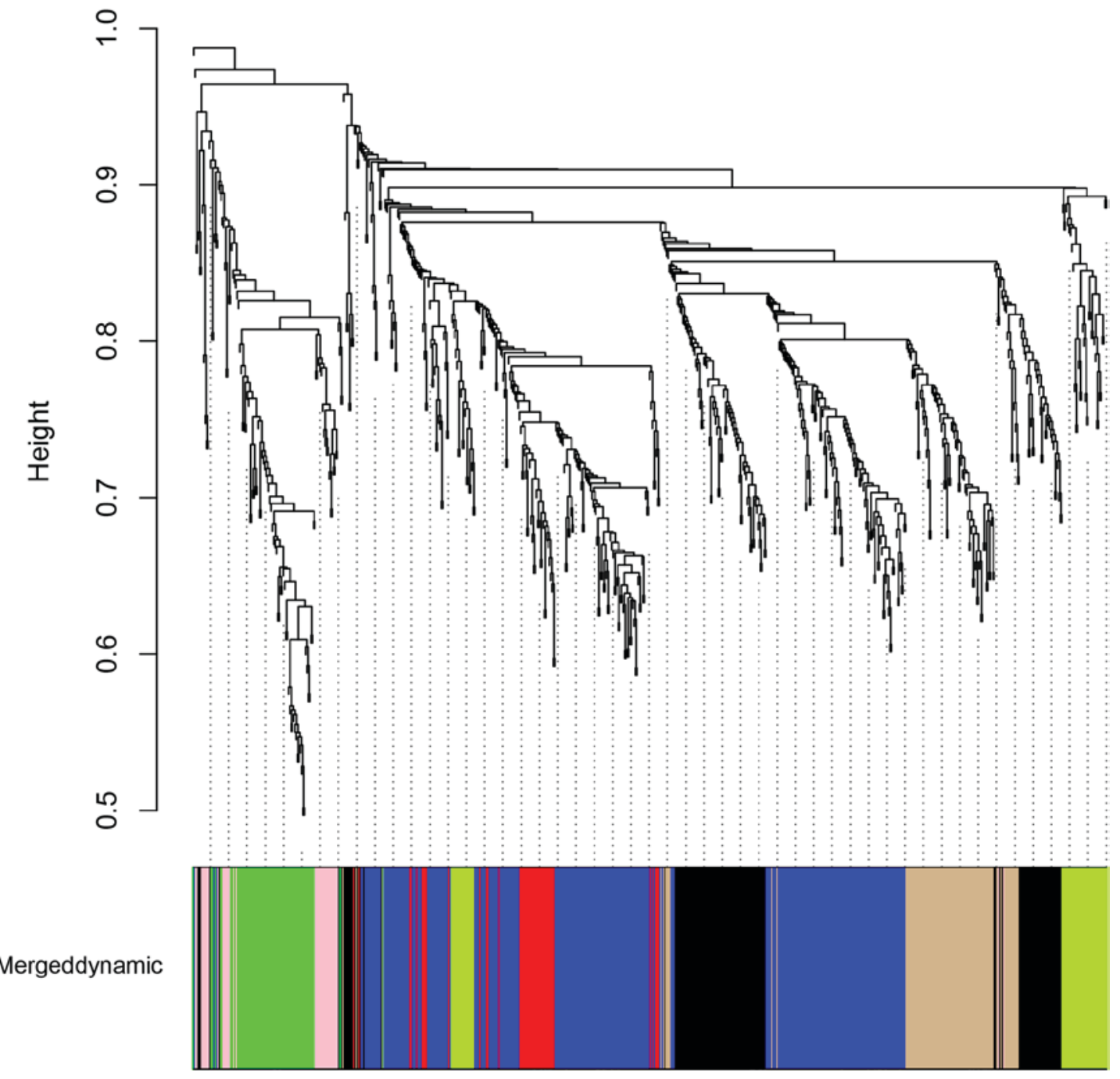

Figure 4. Cluster dendrogram. Seven colors including pink, green/yellow, black, blue, green, red and tan represent seven modules obtained from WGCNA module analyses of DEGs. One color represents one module. The degree of co-expression for the genes in the same modules was high. WGCNA, weighted gene co-expression network analysis. 
Table II. Results of module analysis.

\begin{tabular}{|c|c|c|c|c|c|c|c|}
\hline Factor & $\begin{array}{c}\text { Pink } \\
\text { module }\end{array}$ & $\begin{array}{l}\text { Green/yellow } \\
\text { module }\end{array}$ & $\begin{array}{l}\text { Black } \\
\text { module }\end{array}$ & $\begin{array}{c}\text { Blue } \\
\text { module }\end{array}$ & $\begin{array}{c}\text { Green } \\
\text { module }\end{array}$ & $\begin{array}{c}\text { Red } \\
\text { module }\end{array}$ & $\begin{array}{c}\text { Tan } \\
\text { module }\end{array}$ \\
\hline Gene count & 37 & 53 & 125 & 273 & 69 & 46 & 90 \\
\hline Correlation & $-7.30 \mathrm{E}-01$ & $-1.00 \mathrm{E}-01$ & $-5.80 \mathrm{E}-01$ & $-9.00 \mathrm{E}-02$ & $7.50 \mathrm{E}-01$ & $-7.80 \mathrm{E}-02$ & $2.80 \mathrm{E}-01$ \\
\hline P-value & $5.29 \mathrm{E}-05$ & 6.39E-01 & $3.08 \mathrm{E}-03$ & $6.77 \mathrm{E}-01$ & $2.28 \mathrm{E}-05$ & 7.18E-01 & $1.88 \mathrm{E}-01$ \\
\hline
\end{tabular}

Seven modules (pink, green/yellow, black, blue, green, red and tan) represent modules obtained from WGCNA module analyses of DEGs. One color represents one module. The degree of co-expression for the genes in the same modules was high. WGCNA, Weighted gene co-expression network analysis.

Table III. Functional enrichment results of pink and green modules.

A, Pink module

\begin{tabular}{|c|c|c|c|c|}
\hline GO/KEGG & Term & Description & P-value & Gene \\
\hline \multirow[t]{5}{*}{ GO-BP } & GO:0006631 & Fatty acid metabolic process & $8.00 \mathrm{E}-04$ & Cd36, Mlycd, Acot2, Fabp4, Decr1 \\
\hline & GO:0006936 & Muscle contraction & $9.00 \mathrm{E}-04$ & Tnnt3, Tnnc2, Mylpf, Actn3 \\
\hline & GO:0003012 & Muscle system process & $1.30 \mathrm{E}-03$ & Tnnt3, Tnnc2, Mylpf, Actn3 \\
\hline & GO:0034440 & Lipid oxidation & $3.70 \mathrm{E}-03$ & Cd36, Mlycd, Decr1 \\
\hline & GO:0019395 & Fatty acid oxidation & $3.70 \mathrm{E}-03$ & Cd36, Mlycd, Decr1 \\
\hline \multirow[t]{5}{*}{$\mathrm{GO}-\mathrm{CC}$} & GO:0015629 & Actin cytoskeleton & $9.80 \mathrm{E}-03$ & Tnnt3, Tnnc2, Mylpf, Myoz1 \\
\hline & GO:0005861 & Troponin complex & $1.70 \mathrm{E}-02$ & Tnnt3, Tnnc2 \\
\hline & GO:0030016 & Myofibril & $1.83 \mathrm{E}-02$ & Tnnt3, Tnnc2, Mylpf \\
\hline & GO:0005865 & Striated muscle thin filament & $1.91 \mathrm{E}-02$ & Tnnt3, Tnnc2 \\
\hline & GO:0043292 & Contractile fiber & $2.12 \mathrm{E}-02$ & Tnnt3, Tnnc2, Mylpf \\
\hline \multirow[t]{4}{*}{ GO-MF } & GO:0048037 & Cofactor binding & $1.70 \mathrm{E}-03$ & Ldhb, Nox1, Actn3, Decr1, Nqo1 \\
\hline & GO:0008092 & Cytoskeletal protein binding & $4.30 \mathrm{E}-03$ & Tnnt3, Tnnc2, Mylpf, Myoz1, Actn3 \\
\hline & GO:0050662 & Coenzyme binding & $6.80 \mathrm{E}-03$ & Ldhb, Nox1, Decr1, Nqo1 \\
\hline & GO:0003779 & Actin binding & $7.50 \mathrm{E}-03$ & Tnnt3, Tnnc2, Mylpf, Actn3 \\
\hline \multirow[t]{4}{*}{ KEGG } & rno04510 & Focal adhesion & $1.67 \mathrm{E}-02$ & Col6A3, Mylpf, Actn3, Col5A3 \\
\hline & rno03320 & PPAR signaling pathway & $1.70 \mathrm{E}-02$ & Cd36, Fabp4, Aqp7 \\
\hline & rno04512 & ECM-receptor interaction & $2.18 \mathrm{E}-02$ & Cd36, Col6A3, Col5A3 \\
\hline & rno04670 & $\begin{array}{l}\text { Leukocyte transendothelial } \\
\text { migration }\end{array}$ & $4.17 \mathrm{E}-02$ & Nox1, Mylpf, Actn3 \\
\hline
\end{tabular}

B, Green module

\begin{tabular}{|c|c|c|c|c|}
\hline GO/KEGG & Term & Description & P-value & Gene \\
\hline \multirow[t]{5}{*}{ GO-BP } & GO:0030199 & Collagen fibril organization & $1.00 \mathrm{E}-04$ & Col3A1, Lox, Col5A2, Col5A1 \\
\hline & GO:0030198 & $\begin{array}{l}\text { Extracellular matrix } \\
\text { organization }\end{array}$ & $4.00 \mathrm{E}-04$ & Col3A1, Ccdc80, Lox, Col5A2, Col5A1 \\
\hline & GO:0042060 & Wound healing & $6.00 \mathrm{E}-04$ & Ccnb1, Casp3, Col3A1, Lox, Tfpi2, Col5A1 \\
\hline & GO:0030155 & Regulation of cell adhesion & $1.30 \mathrm{E}-03$ & Tnc, Ccdc80, Jak2, Mmp14, Col8A1 \\
\hline & GO:0007049 & Cell cycle & $2.10 \mathrm{E}-03$ & $\begin{array}{l}\text { Ccnb1, Ccnb2, Mki67, Cks2, Pttg1, Cdkn3, } \\
\text { Ube2C, Racgap1 }\end{array}$ \\
\hline \multirow[t]{2}{*}{$\mathrm{GO}-\mathrm{CC}$} & GO:0031012 & Extracellular matrix & $6.06 \mathrm{E}-14$ & $\begin{array}{l}\text { Matn2, Aspn, Cthrc1, Tnc, Col3A1, Ccdc80, } \\
\text { Mmp14, Col5A2, Col5A1, Col6A2, Col6A1, } \\
\text { Lox, Lox11, Thbs4, Adamts4, Spon1 }\end{array}$ \\
\hline & GO:0005578 & $\begin{array}{l}\text { Proteinaceous extracellular } \\
\text { matrix }\end{array}$ & $4.22 \mathrm{E}-12$ & $\begin{array}{l}\text { Matn2, Aspn, Cthrc1, Tnc, Col3A1, Ccdc80, } \\
\text { Lox, Mmp14, Col5A2, Lox11, Col5A1, } \\
\text { Adamts4, Spon1, Thbs4 }\end{array}$ \\
\hline
\end{tabular}


Table III. Continued.

\begin{tabular}{|c|c|c|c|c|}
\hline GO/KEGG & Term & Description & P-value & Gene \\
\hline & GO:0044421 & Extracellular region part & $1.36 \mathrm{E}-09$ & $\begin{array}{l}\text { Matn2, Aspn, Cthrc1, Tnc, Col3A1, Ccdc80, } \\
\text { Mmp14, Col5A2, Col5A1, Ctsk, Cpxm1, } \\
\text { Col6A2, Col6A1, Lox, Loxl1, Thbs4, Adamts4 } \\
\text { Spon1 }\end{array}$ \\
\hline & GO:0005576 & Extracellular region & $1.18 \mathrm{E}-08$ & $\begin{array}{l}\text { Matn2, Aspn, Cthrc1, Aebp1, Tnc, Col3A1, } \\
\text { Ccdc80, Mmp14, Col5A2, Col5A1, Ctsk, Penk, } \\
\text { Cpxm1, Sfrp4, Col6A2, Co16A1, Lox, Loxl1, } \\
\text { Tfpi2, Thbs4, Adamts4, Spon1 }\end{array}$ \\
\hline & GO:0005581 & Collagen & $9.18 \mathrm{E}-05$ & Col3A1, Lox, Col5A2, Col5A1 \\
\hline \multirow[t]{5}{*}{ GO-MF } & GO:0016641 & $\begin{array}{l}\text { Oxidoreductase activity, acting } \\
\text { on the } \mathrm{CH}-\mathrm{NH} 2 \text { group of donors, } \\
\text { oxygen as acceptor }\end{array}$ & $1.50 \mathrm{E}-03$ & Lox, Lox12, Lox11 \\
\hline & GO:0016638 & $\begin{array}{l}\text { Oxidoreductase activity, acting } \\
\text { on the } \mathrm{CH}-\mathrm{NH} 2 \text { group of donors }\end{array}$ & $2.20 \mathrm{E}-03$ & Lox, Lox12, Lox11 \\
\hline & GO:0005201 & $\begin{array}{l}\text { Extracellular matrix structural } \\
\text { constituent }\end{array}$ & $5.20 \mathrm{E}-03$ & Col3A1, Col5A2, Col5A1 \\
\hline & GO:0016702 & $\begin{array}{l}\text { Oxidoreductase activity, acting } \\
\text { on single donors with incorporation } \\
\text { of molecular oxygen, incorporation } \\
\text { of two atoms of oxygen }\end{array}$ & $6.40 \mathrm{E}-03$ & Plod2, P4Ha3, Hpd \\
\hline & GO:0016701 & $\begin{array}{l}\text { Oxidoreductase activity, acting on } \\
\text { single donors with incorporation } \\
\text { of molecular oxygen }\end{array}$ & $6.80 \mathrm{E}-03$ & Plod2, P4Ha3, Hpd \\
\hline \multirow[t]{3}{*}{ KEGG } & rno04512 & ECM-receptor interaction & $6.37 \mathrm{E}-07$ & $\begin{array}{l}\text { Tnc, Col3A1, Col6A2, Col6A1, Col5A2, } \\
\text { Col5A1, Thbs4 }\end{array}$ \\
\hline & rno04510 & Focal adhesion & $1.03 \mathrm{E}-04$ & $\begin{array}{l}\text { Tnc, Col3A1, Col6A2, Col6A1, Col5A2, } \\
\text { Col5A1, Thbs4 }\end{array}$ \\
\hline & rno04115 & p53 signaling pathway & $2.96 \mathrm{E}-02$ & Ccnb1, Casp3, Ccnb2 \\
\hline
\end{tabular}

GO, gene ontology; KEGG, Kyoto Encyclopedia of Genes and Genomes; BP, biological process; CC, cellular component; MF, molecular function.

modules are summarized in Table III. It was demonstrated that the PPAR signaling pathway that cluster of differentiation 36 (CD36) was significantly enriched in, was one of the significant pathways in the pink module. In addition, the p53 signaling pathway, that caspase-3 (Casp3) was significantly enriched in, was one of the significant pathways in the green module (Table III).

\section{Discussion}

In the present study, a total of 693 genes were identified by combining the DEGs of the SCI3d, SCI8d and SCI14d groups. The results obtained demonstrated that the PPAR signaling pathway, in which CD36 was significantly enriched, was one of the significant pathways in the pink module, while the p53 signaling pathway that Casp3 was significantly enriched in, was one of the significant pathways in the green module.

PPAR, which includes 3 isoforms (PPAR- $\alpha$ PPAR- $\gamma$ PPAR- $\beta / \delta)$, is involved in the inflammation process (22). A previous study demonstrated that PPAR $-\gamma$ and PPAR- $\delta$ are involved in the protective effects of palmitoylethanolamide (PEA) in SCI, indicating that PPAR $-\gamma$ and PPAR- $\delta$ may contribute to the anti-inflammatory effects of PEA in SCI (23). It has been reported that PPAR participates in the pathogenesis of diseases, such as diabetes and SCI $(24,25)$. In addition, it has been observed that the decrease of PPAR- $\delta$ expression in the spinal cord of streptozotocin (STZ)-diabetic rats may explain the higher mortality rate observed following SCI in STZ-diabetic rats (26). Thus, activation of PPAR- $\delta$ may reduce the severity of SCI, and PPAR- $\delta$ may be a target for therapy in SCI patients (27). In addition, van Neerven and Mey (28) indicated that endogenous PPAR ligands may contribute by preventing the expansion of the initial damage via modulating inflammation post-SCI, and concluded that the PPAR signaling pathway may be a target for treatment of SCI. Therefore, the results of the present study are in agreement with the results of previous studies and, as such, the PPAR signaling pathway may be closely associated with the pathogenesis of SCI. In addition, the results demonstrated that $C D 36$ was significantly enriched in the PPAR signaling pathway. It has been indicated that the 
upregulation of $C D 36$, an integral membrane protein, may resolve inflammation via the 5-lipoxygenase-dependent and PPAR- $\gamma$ signaling pathways (29). The wnt-1 proto-oncogene protein promotes $C D 36$ expression via $\beta$-catenin and PPAR- $\gamma$ signaling pathways (30). CD36 is involved in the PPAR- $\gamma$ signaling pathway, and activation of PPAR- $\gamma$ leads to upregulation of $C D 36$ in the PPAR signaling pathway (31). Previous studies have indicated that CD36 is involved in the PPAR- $\gamma$ signaling pathway $(22,32)$. Although the roles of CD36 in SCI have not been extensively studied, when considering the association between the PPAR signaling pathway and SCI, it is possible that CD36 may be involved in the progression of SCI via the PPAR signaling pathway.

The p53 signaling pathway was observed to be one of the significant KEGG pathways in the green module in the present study. A previous study observed DNA damage-induced p53-mediated mitochondrial apoptosis in SCI (5). Nerve injury is a significant consequence of SCI, and p53 is involved in glial cell death and survival in SCI (33). p53, a key molecular regulator of apoptotic cell death, is known to promote apoptosis by increasing the transcription of target genes $(34,35)$. In addition, a number of apoptosis-associated molecules are expressed via p53, and apoptosis induction via the p53 pathway is a complex process (33). Furthermore, minocycline has been shown to reduce apoptosis in models of SCI (36). Therefore, the results of the present study are consistent with previous studies, and therefore indicate that the p53 signaling pathway may be significant in the progression of SCI. In the present study, it was demonstrated that Casp3 was significantly enriched in the p53 signaling pathway. It has been observed that Casp3 and the p53 signaling pathway may regulate nitric oxide-induced extracellular signal-regulated protein kinase and p38 kinase (37). In addition, p53 prevents the $\alpha 6 \beta 4$ integrin-mediated activation of serine/threonine-specific protein kinase B by promoting Casp3 dependent cleavage (38). It has been suggested that Casp3 is a critical mediator of p53-induced apoptosis (39). Zhang et al (40) investigated the apoptosis process in SCI, and demonstrated that the Casp3 apoptotic pathway components are activated following SCI in rats. Ultimately, it is thought that Casp3 may be involved in the progression of SCI via the p53 signaling pathway.

In conclusion, the PPAR and p53 signaling pathways may be important pathways associated with SCI. In addition, CD36 and Casp3 may be involved in the progression of SCI via the PPAR and p53 signaling pathways, respectively. However, the results of the present study are limited by the small sample size, and therefore further studies are required to evaluate the molecular mechanisms underlying SCI progression.

\section{Acknowledgements}

The present study was supported by the National Natural Science Foundation of China (grant no. 81472120) and the Science and Technology Commission of Shanghai Municipality (grant no. 14140903900).

\section{References}

1. Shah RR and Tisherman SA: Spinal Cord Injury. Springer London, 2014.
2. Me VDB, Castellote JM, Mahillo-Fernandez I and De P-CJ: Incidence of spinal cord injury worldwide: A systematic review. Neuroepidemiology 34: 184-192, 2010.

3. Sekhon LH and Fehlings MG: Epidemiology, demographics, and pathophysiology of acute spinal cord injury. Spine (Phila Pa 1976) 26 (24 Suppl): S2-S12, 2001.

4. Wyndaele M and Wyndaele JJ: Incidence, prevalence and epidemiology of spinal cord injury: What learns a worldwide literature survey? Spinal Cord 44: 523-529, 2006.

5. Kotipatruni RR, Dasari VR, Veeravalli KK, Dinh DH, Fassett D and Rao JS: p53- and bax-mediated apoptosis in injured rat spinal cord. Neurochem Res 36: 2063-2074, 2011.

6. Wanner IB, Anderson MA, Song B, Levine J, Fernandez A, Gray-Thompson Z, Ao Y and Sofroniew MV: Glial scar borders are formed by newly proliferated, elongated astrocytes that interact to corral inflammatory and fibrotic cells via STAT3-dependent mechanisms after spinal cord injury. J Neurosci 33: 12870-12886, 2013.

7. Tachibana T, Noguchi K and Ruda MA: Analysis of gene expression following spinal cord injury in rat using complementary DNA microarray. Neurosci Lett 327: 133-137, 2002.

8. Bastien D, Bellver Landete V, Lessard M, Vallières $N$, Champagne M, Takashima A, Tremblay MÈ, Doyon Y and Lacroix S: IL-1 $\alpha$ gene deletion protects oligodendrocytes after spinal cord injury through upregulation of the survival factor Tox3. J Neurosci 35: 10715-10730, 2015.

9. Munro KM, Perreau VM and Turnley AM: Differential gene expression in the EphA4 knockout spinal cord and analysis of the inflammatory response following spinal cord injury. PLoS One 7: e37635, 2011.

10. Guerrero AR, Uchida K, Nakajima H, Watanabe S, Nakamura M, Johnson WE and Baba H: Blockade of interleukin-6 signaling inhibits the classic pathway and promotes an alternative pathway of macrophage activation after spinal cord injury in mice. J Neuroinflammation 9: 40, 2012.

11. Barrett T, Wilhite SE, Ledoux P, Evangelista C, Kim IF, Tomashevsky M, Marshall KA, Phillippy KH, Sherman PM, Holko M, et al: NCBI GEO: Archive for functional genomics data sets-update. Nucleic Acids Res 41 (Database issue): D991-D995, 2013.

12. Gautier L, Cope L, Bolstad BM and Irizarry RA: Affy-analysis of Affymetrix GeneChip data at the probe level. Bioinformatics 20 : 307-315, 2004.

13. Ritchie ME, Phipson B, Wu D, Hu Y, Law CW, Shi W and Smyth GK: limma powers differential expression analyses for RNA-sequencing and microarray studies. Nucleic Acids Res 43: e47, 2015 .

14. Smyth GK: Linear models and empirical bayes methods for assessing differential expression in microarray experiments. Stat Appl Genet Mol Biol 3: Article3, 2004.

15. Ferreira JA: The Benjamini-Hochberg method in the case of discrete test statistics. Int J Biostat 3: Article 11, 2007.

16. Warnes GR, Bolker B, Bonebakker L, Gentleman R, Huber W, Liaw A, Lumley T, Maechler M, Magnusson A, Moeller S, et al: gplots: Various $\mathrm{R}$ programming tools for plotting data. R package version 2, 2005.

17. Oliveros JC: VENNY. An interactive tool for comparing lists with Venn Diagrams, 2007.

18. Altermann E and Klaenhammer TR: PathwayVoyager: Pathway mapping using the kyoto encyclopedia of genes and genomes (KEGG) database. BMC Genom 6: 203-213, 2005.

19. Huang DW, Sherman BT and Lempicki RA: Systematic and integrative analysis of large gene lists using DAVID bioinformatics resources. Nature Protoc 4: 44-57, 2008.

20. Langfelder $P$ and Horvath S: WGCNA: An R package for weighted correlation network analysis. BMC Bioinformatics 9: 559, 2008.

21. Ashburner M, Ball CA, Blake JA, Botstein D, Butler H, Cherry JM, Davis AP, Dolinski K, Dwight SS, Eppig JT, et al: Gene Ontology: Tool for the unification of biology. The Gene Ontology Consortium. Nat Genet 25: 25-29, 2000.

22. Yamanaka M, Ishikawa T, Griep A, Axt D, Kummer MP and Heneka MT: PPAR $\gamma /$ RXR $\alpha$-induced and CD36-mediated microglial amyloid- $\beta$ phagocytosis results in cognitive improvement in amyloid precursor protein/presenilin 1 mice. J Neurosci 32: 17321-17331, 2012.

23. Paterniti I, Impellizzeri D, Crupi R, Morabito R, Campolo M, Esposito E and Cuzzocrea S: Molecular evidence for the involvement of PPAR- $\delta$ and PPAR- $\gamma$ in anti-inflammatory and neuroprotective activities of palmitoylethanolamide after spinal cord trauma. J Neuroinflammation 10: 20, 2013. 
24. Murphy GJ and Holder JC: PPAR- $\gamma$ agonists: Therapeutic role in diabetes, inflammation and cancer. Trends Pharmacol Sci 21: 469-474, 2000.

25. McTigue DM: Potential therapeutic targets for PPAR gamma after Spinal Cord Injury. PPAR Res 2008: 517162, 2008.

26. Tsai CC, Lee KS, Chen SH, Chen LJ, Liu KF and Cheng JT: Decrease of PPARd in type-1-like diabetic rat for higher mortality after spinal cord injury. PPAR Res 2014: 456386, 2014

27. Esposito E and Cuzzocrea S: Targeting the peroxisome proliferator-activated receptors (PPARs) in spinal cord injury. Expert Opin Ther Targets 15: 943-959, 2011.

28. van Neerven $S$ and Mey J: RAR/RXR and PPAR/RXR signaling in spinal cord injury. PPAR Res 2007: 29275, 2007.

29. Ballesteros I, Cuartero MI, Pradillo JM, de la Parra J, Pérez-Ruiz A, Corbí A, Ricote M, Hamilton JA, Sobrado M, Vivancos J, et al: Rosiglitazone-induced CD36 up-regulation resolves inflammation by PPAR $\gamma$ and 5-LO-dependent pathways. J Leukoc Biol 95: 587-598, 2014.

30. Yan H, Wang S, Chen T and Zhu J: oxLDL decreases wnt1 which promotes CD36 through b-catenin and PPAR-r signaling pathway in macrophage. In: European heart journal Oxford Univ Press Great Clarendon St, Oxford OX2 6DP, England, pp1123-1123, 2014.

31. Nagaraj S, Raghavan AV, Rao SN and Manjappara UV: Obestatin and Nt8U influence glycerolipid metabolism and PPAR gamma signaling in mice. Int J Biochem Cell Biol 53: 414-422, 2014.

32. Lourenco MV and Ledo JH: Targeting Alzheimer's pathology through PPAR $\gamma$ signaling: Modulation of microglial function. J Neurosci 33: 5083-5084, 2013.
33. Saito N, Yamamoto T, Watanabe T, Abe Y and Kumagai T: Implications of $\mathrm{p} 53$ protein expression in experimental spinal cord injury. J Neurotrauma 17: 173-182, 2000.

34. Fridman JS and Lowe SW: Control of apoptosis by p53. Oncogene 22: 9030-9040, 2003.

35. Slee EA, O'Connor DJ and Lu X: To die or not to die: How does p53 decide? Oncogene 23: 2809-2818, 2004.

36. Teng YD, Choi H, Onario RC, Zhu S, Desilets FC, Lan S, Woodard EJ, Snyder EY, Eichler ME and Friedlander RM: Minocycline inhibits contusion-triggered mitochondrial cytochrome $\mathrm{c}$ release and mitigates functional deficits after spinal cord injury. Proc Natl Acad Sci USA 101: 3071-3076, 2004.

37. Kim SJ, Ju JW, Oh CD, Yoon YM, Song WK, Kim JH, Yoo YJ, Bang OS, Kang SS and Chun JS: ERK-1/2 and p38 kinase oppositely regulate nitric oxide-induced apoptosis of chondrocytes in association with $\mathrm{p} 53$, caspase-3, and differentiation status. J Biol Chem 277: 1332-1339, 2002.

38. Bachelder RE, Ribick MJ, Marchetti A, Falcioni R, Soddu S, Davis KR and Mercurio AM: p53 inhibits alpha 6 beta 4 integrin survival signaling by promoting the caspase 3 -dependent cleavage of AKT/PKB. J Cell Biol 147: 1063-1072, 1999.

39. Communal C, Sumandea M, De Tombe P, Narula J, Solaro RJ and Hajjar RJ: Functional consequences of caspase activation in cardiac myocytes. Proc Natl Acad Sci USA 99: 6252-6256, 2002.

40. Zhang N, Yin Y, Xu SJ, Wu YP and Chen WS: Inflammation \& apoptosis in spinal cord injury. Indian J Med Res 135: 287-296, 2012. 